INTERNATIONAL DESIGN CONFERENCE - DESIGN 2018

https://doi.org/10.21278/idc.2018.0468

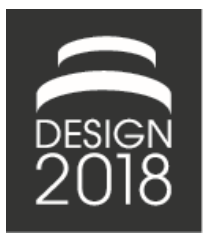

\title{
REAL-TIME CODING METHOD FOR CAPTURE OF ARTEFACT-CENTRIC INTERACTIONS IN CO- CREATIVE DESIGN SESSIONS
}

\author{
F. Ben Guefrache, C. Masclet, G. Prudhomme, G. Cascini and J. A. O'Hare
}

\begin{abstract}
This paper proposes a method to speed up the quantitative description of interaction occurrences during co-creative design sessions. Protocol analysis is traditionally used for such studies but it requires capturing video and cumbersome post session analyzing - making it too time consuming for analyzing a large number of sessions. We developed a new 'on the fly' method based on direct observation and compared the results obtained by this method to those obtained with traditional post-session coding method. Results were found to be very similar, whilst analysis time was significantly reduced.
\end{abstract}

Keywords: co-design, design research methodology, protocol analysis, augmented reality $(A R)$

\section{Introduction}

Protocol analysis has been used extensively to study individual or collective aspects of designing, especially since the early 90s (Cross et al., 1996). One of the main drawback of protocol analysis methodologies relies on the post session analysis of the data. The data analysis carried out by the researchers is following a long and cumbersome process. Therefore, it is impossible to conduct a big number of design experiments that allow comparative analysis with quantitative data and results. This is why we consider there is a need for a real time coding method. We understand this approach as complementary to a classical post-session data analysis. It can bring quick quantitative results on some key parameters. Furthermore, the ultimate goal is to be able to link performance indicators with gesture patterns, which can inform the design of interaction devices. This paper proposes a step ahead towards a real-time coding method providing to a quick and reliable results with the least possible resources.

We address two research questions:

- Is it possible to create an on the fly observation method, that allows real time capture of primary data sets?

- Is this approach reliable so that we can skip a post session analysis of these primary data?

This approach is motivated by the necessity to analyse a big amount of data that will be captured in the context of the SPARK European project. The SPARK project (http://spark-project.net/), funded by the European Union's Horizon 2020 Research and Innovation program, aims at facilitating the co-creative interactions within collaborative design sessions involving end-users by providing a responsive ICT platform based on Spatial Augmented Reality (SAR) that allows creating, visualizing, assessing and modifying design concepts since the earliest stages of a design process. The goal is reducing language and cognitive barriers due to diversity of nationalities, backgrounds and motivations of the end-users. 
Indeed, one of the most important trends in today's information and communication society is the fact that more and more people do not only use products but also want to be active in the understanding, modification or even creation of these products (Ardito et al., 2012). The participation of end users in the early design phases is critical and implies the need for evolution of the processes, tools and practices. This collaborative aspect is about working together in design settings with the aim of taking advantage of what Steiner calls process gain (Thomas, 2000). A complementary vision of end-users associated to the skills of the designers brings to the success of a design users-centred task.

Related to the SPARK objectives, our method focusses on artefact-centric interactions analysis and aims to quantify the distribution of these interactions between end-users and designers as an indicator of the collaboration pattern of each co-creative session. The main purpose of this work is to analyse the influence of artefacts used within a co-creative session. Our analyses are based on live observations of design experiments, which involved real professional creative designers and end-users. These observations were held in collaboration with our industrial partners: two design companies.

The next section (section 2) presents a brief overview of methodologies used for speech and gesture analyses of collaborative design sessions with the aim of highlighting what is already established in the design community and underline the gap we are addressing in this paper. Section 3 presents the 'on the fly' method and the way we built it. In section 4, results from validation of this method are addressed through the comparison of on the fly coding and post session coding verifications. Then we will conclude in section 5 .

\section{State of the art: From classical post-session coding to on the fly coding}

Protocol analysis is a well-known research method that is recognised as rigorous and has been used extensively to analyse and understand design, and more specifically how designers think and behave individually or collectively while they are designing. Most of the research effort focuses on verbal and visual cognition and surprisingly limited attention is paid to gestures as noticed by (Kendira et al., 2011). However, design interactions occur through different modalities: verbal, graphical, gestural, and other modalities (gaze, posture, prosody), which is considered as visual data (sketching or gesture) and generated by designers as an extension to the problem-solving process (Davis, 2016).

\subsection{Speech interaction analysis methodology}

The domain of design protocol studies refers generally to analysis of spoken interactions among participants in collaborative sessions. We exclude here design studies that used variants of the thinkaloud techniques (Ericsson and Simon, 1993). Speech information are composed by utterances, which contain information related to the assessment and performance of actions, or, as reported by Austin "things that people do with words" (Austin, 1962). Hay et al. (2016) confirm that design protocol analysis mainly aims at capturing relevant cognitive processes in order to characterise the design activity. Whatever the goal of protocol studies is, the methodological process follows a standard procedure: after the definition of a coding scheme to characterise what happens during the design sessions, the verbal interactions of designers are recorded and transcribed. Then, the transcription of the design session is segmented and classified according to the coding scheme, which makes the data ready for the analysis and the extraction of relevant conclusions (Gero and Mc Neill, 1998): this is what we call later post-processing. This process is very effective for a deep analysis of designers' behaviour and cognition, including visual cognition. In the following section, we propose some refinements to this process to consider gesture analysis and the role of artefacts in design cognition.

\subsection{Gestural interaction analysis methodology}

Gestures in design have been examined from diverse perspectives leading to different categorisation of gesture interactions. However, it is most generally supposed to play a role in communication. The International Society for Gesture Studies (www.gesturestudies.com) states that gesture studies are concerned with how people use their hands and other parts of their body for communicative purposes. The gesture specialist David McNeill (McNeill, 1992) identifies four categories of gesture; i.e. iconic, metaphoric, deictic (pointing), and beat gestures. McNeill's research has identified links between 
gesture, speech and thought that, despite the strong connection that gestures have with speech, are fundamentally different in form compared to speech. McNeill suggests that some dimensions of thought are embedded in the gesture. These findings have implications on the role of gesture in designing: the role it plays in design thinking and the role it plays in design collaboration as being more than simple communication vehicle (Visser and Maher, 2011). Following Visser (2010), the function of gesture can be distinguished by two types: Interactive and other. Interactive gesture is used to manage interactions between the different participants in a design meeting. Other gestures are used to generate and evaluate design proposals. It also can play a role in Computer-supported design environments, allowing designers to transfer their ideas into a digital model. Cash and Maier (2016) highlight how gestural sequences are used to support kinaesthetic thinking and how designers discuss, instigate, and virtually prototype ideas with their hands. The study of Cash and Snider (2014) underlines two factors that describe gestures: the target, which is the interest of the participant's activity and the type.

Most of the cited studies refer to the traditional methodology of capturing videos and audio data. The analysis requires a post-processing phase (as we described in section 2.1) to allow a detailed treatment before conclusions can be drawn.

\subsection{Artefact-centric interaction analysis methodologies}

The literature study showed that gesture analysis has been at the centre of several studies. However, no quantitative approach of design gestures has been provided. Therefore, we found the necessity to develop a method that could fit our requirement for repeatability and robustness. We created our own analysis coding scheme that we tested during three design sessions with professional design teams (Becattini et al., 2017). It is based on three elements: the client(s), the designer(s) and the artefact(s) used to support their interactions with two groups of interactions: with artefacts (Tangible, Digital, Mixed and Ephemeral) and without artefact (None) (Figure 1).

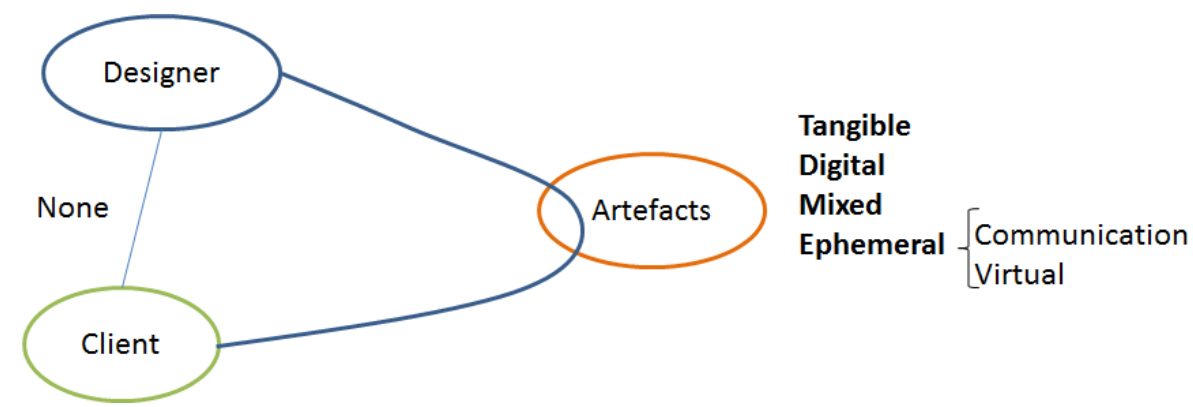

Figure 1. Interaction analysis framework and gestures coding scheme

In this context, the Digital artefacts category (Figure 1) includes any kind of representation displayed on a screen, laptop, and the Tangible artefact category includes physical mock-ups, printed sheets of the alternatives drawings, sticky-notes that will be posted on the white board, or personal notes. The Mixed artefact category is defined as a physical prototype (physical mock-up with a predefined shape, mostly $3 \mathrm{D}$ printed) on which are projected digital elements. They can be pictures, images, text or textures. This is the kind of artefact which are displayed by the SAR (Spatial Augmented Reality) system. Ephemeral artefacts embrace virtual artefacts and communication gestures. Communication gestures correspond to gesturing with hands instead of or while speaking. These hand gestures, beat gesturing (Eris et al., 2014), are complementing speech and are not task specific with some symbolic gestures. A virtual artefact is defined as an imaginary object that is depicted or mimicked by a gesture in the air (Becattini et al., 2017). The person making the gesture can depict or mimic an object (shape, volume, surface), a usage (function in a specific context) or a behaviour (deformation of an object, simulate flashing lights etc.). Given the role gestures play in the designing activity and considering all the studies mentioned, it seems quite reasonable to capture and analyse gesture interactions between participants during design session as an observable phenomenon of the collaborative activity.

From a methodology point of view, our preliminary study was based on three co-design sessions in a real context (in-situ) dedicated to the design and evaluation of three different products. Each session 
was video recorded. This allowed for post session access to artefact-centric gestures, speech and material produced during the sessions. The preliminary results showed very interesting characteristics. Unfortunately, it was not possible to reproduce the conditions and analyse the results with a larger sample size due to the significant amount of time that is required to analyse each session. Qualitative analysis is possible to do but it is a time-consuming procedure. Quantitative analysis is still a timeintensive task due to the inherent structure of our analysis method. Therefore, no conclusions could be drawn concerning the role of SAR artefacts on the performance of the session.

\subsection{On the fly coding methodology}

Our bibliography search did not reveal studies based on any on-the-fly coding to study design collaboration. This is not very surprising as this type of coding is very demanding and hard to assess. However, regarding our requirements of repeating and dealing with a great number of design situations, it seems reasonable to search in that direction. Therefore, we have designed and validated a method that allows capturing and rapidly delivering data from design sessions showing the relative involvement of designers and clients and the distribution of the type of interactions. This method will be presented and its validity discussed hereafter.

\section{On the fly coding method to capture gesture interactions}

\subsection{Description of the method}

What do we expect to obtain at the end of the method application? The objective is to reach a quantitative description of gestural artefact-centric interactions occurrences made by designers and clients during a specific co-creative design session. These interactions (that we call 'events' hereafter) are considered (and captured) only when they occur simultaneously with a spoken utterance. Talking is our indicator to detect a collaborative activity.

Our method is divided in two steps:

- Coding step: the objective of the first coding step is to obtain primary data, doing a coding on the fly during the session. This is done through direct observation with two coders being present during the sessions.

- Analysis step: The second one is to process and analyse these primary data with the objective to get a quantitative description of the interaction occurrences of the sessions.

The coding step has to be made in real time by two coders using a tool we named 'Observer'. One coder has to code who intervenes, more precisely who is at the origin of the collaborative interaction: Client or Designer. The second coder has to code types of artefact that are supporting interactions between clients and designers. Interfaces proposed to coders are described Figure 2 and 3.

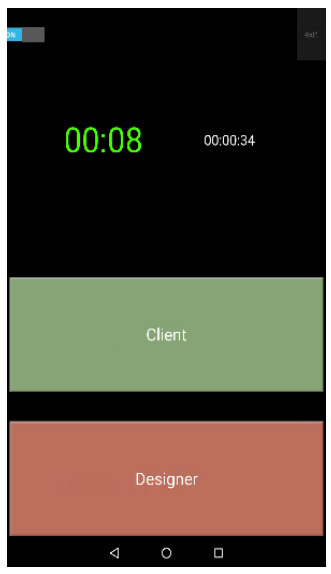

Figure 2. Interface for coding actors during interactions

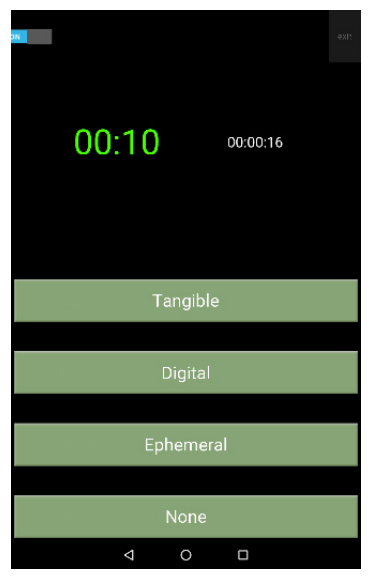

Figure 3. Interface for coding the artefact supporting interactions 
A coding book (including rules for coding actors and artefacts) has been developed, which can be used as a support for training session for novice coders or as a reminder before each new session for more expert coders.

The analysis step is currently partially automated by means of a software program that transforms the separated coding results provided by each coder into a first draft of merged result. Figure $4 \mathrm{a}$ and $4 \mathrm{~b}$ shows excerpts of the coders' tables including timestamps of recorded events associated to the nature of the event in terms of 1) occurrence of actor's intervention and 2) artefact-centric interaction.

Figure $4 \mathrm{c}$ shows an excerpt of the merged table based on a timeline incremented every second (first column), the occurrence of actors intervening at this time if it is the case (column 2), and the occurrence of artefacts supporting the interaction if there is one at this time.

\begin{tabular}{|c|c|c|c|c|c|c|c|c|}
\hline & & $00: 05: 41$ & Designer & Ephemeral & 00:05:41 & Designer & Ephemeral & D Ephemeral \\
\hline & & $00: 05: 42$ & Designer & Ephemeral & 00:05:42 & Designer & Ephemeral & D Ephemeral \\
\hline & 00:05:41 Ephemeral & $00: 05: 43$ & Digital & & 00:05:43 & & Digital & \\
\hline & 00:05:42 Ephemeral & $00: 05: 44$ & Client & & 00:05:44 & Client & & C Digital \\
\hline 00:05:41 Designer & 00:05:43 Digital & $00: 05: 45$ & & & 00:05:45 & & & \\
\hline 00:05:42 Designer & 00:05:46 Ephemeral & $00: 05: 46$ & Ephemeral & & 00:05:46 & & Ephemeral & C Ephemeral \\
\hline 00:05:44 Client & 00:05:47 Mixed & $00: 05: 47$ & Mixed & & 00:05:47 & & Mixed & C Mixed \\
\hline 00:05:49 Designer & 00:05:49 Ephemeral & $00: 05: 48$ & & & 00:05:48 & & & \\
\hline & 00:05:51 Mixed & $00: 05: 49$ & Designer & Ephemeral & 00:05:49 & Designer & Ephemeral & D Ephemeral \\
\hline & & $00: 05: 50$ & & & 00:05:50 & & & \\
\hline & & $00: 05: 51$ & Mixed & & 00:05:51 & & Mixed & D Mixed \\
\hline
\end{tabular}

Figure 4. From left to right: Actor table (a), Artefact table (b), Automatized Merged Table (c), final result (d)

At this time, the final merging of the results (Figure 4d) obtained from the automatized proposal is made manually. The way to get it will be explained in the following section. A 'set of rules' has been defined to operate the merging when some decisions, context dependent, require a human processing.

\subsection{Rational for building the method}

The method is dedicated to the observation of interactions that are carried out in a continuous flow. Interactions are characterized by the nature (type of) of the artefact manipulated and the actor (type of actor) who is initiating the interaction. See section 2.3 for more details.

\subsubsection{Defining the Observer tool structure}

The two steps of the method we proposed (section 3.1) are strongly interrelated. The difficulty of the analysis step is strongly dependent of the way the coding step has been done. The more complete the first one is the easiest the second one will be. Straight from our coding scheme, we defined, in a first attempt, a Graphical User Interface (GUI) which proposed eleven digital buttons: ten buttons associating the combination of the actors (designers or clients) with the type of artefacts (five types); the eleventh button was intended to be used when the coder was not able to code (too many events happening in a short time for instance) or to mark an unexpected event, not pertaining to the coding scheme (technical problem for example). One coder was initially supposed to code all interactions. However, different tests demonstrated that such a proposition was not cognitively acceptable and that the coder was unable to sustain the coding during a full session (which, in our case, can last up to $1 \mathrm{~h} 30$ ). Furthermore, playing with the eleven buttons was not easy, even after a long time of learning.

Due to this task complexity and the cognitive load of the coder(s), we decided to split the coding process in two separate parts, to be completed by two coders. According to the project constraints (real work situations, non-intrusive observations), a maximum of two coders were allowed to be present in the session room for coding on the fly. This was necessary to keep the non-intrusive criteria to the session observed.

Then, two main criteria led our thinking about the strategy of coding division. Coders must be able to witness every event that has to be recorded. Otherwise the corpus will miss some potentially important events. This means that the event has to be observable. This is usually ensured when the event has clues that can be easily observed in the considered situation: primarily visual and audio clues. Once the event 
has been noticed by the observer, it still has to be classified in the relevant category. Categories have to be simple enough and not too numerous to allow coder to select them on the fly.

Among different modalities we chose to entrust the coding of the interactions initiated by actors to one coder and the related artefacts object of the interactions. This choice is justified by the fact that it could be easier for a human coder to be concentrated:

- On one hand, the actors who intervene, using the voice (audio clue);

- On the other hand, the main support of this interaction, using the gesture clue.

We used a tablet for coding on the fly, and we designed a program with a GUI showing buttons with the events to be tracked down (see Figures 2 and 3). Once the Observer program is launched, the users simultaneously start the recording by pressing the button in the upper left portion of the screen. They can then begin coding individually, pressing the relevant event button each time they witness an event from the coding scheme. The number of buttons is kept to a minimum and allows manipulation with one finger (single hand or both). The main concern was to allow the observer to keep eyes on the scene of the design activity, rather than staring at the tablet in search of the right button.

\subsubsection{Usability testing}

A usability test was conducted, in order to verify that:

- it is possible (in a cognitive way) to code using this Observer tool (i.e. the coder is able to follow the stream of interactions and track down the events),

- Two encoders obtain comparable results (robustness) when coding the same session.

To run this test, two researchers were invited to code, using the Observer tool (tablets), on different excerpts of previously captured sessions which were available in video format. Researchers were then standing in front of a computer, with their tablet, and had to code the session displayed on the screen, without any control on the stream (real time play). For each sample, each one of the coders coded first the actors who intervene (using interface Figure 2) and, in a second time, coded the artefacts that support the interaction (using interface Figure 3).

A first result from this test was that Observer is usable easily for coding the initiating actor. It is also usable for coding artefact, although more effort is required because of the five types of artefact. A second conclusion was that two researchers coding three samples of different sessions lead to a good convergence on the results obtained. We decided to compare these results using Cohen's Kappa index. This allowed us to measure inter-coder agreement, to identify and discuss the disagreements in the coding, and to compile examples of coding difficulties and the final decision that was taken on how to code these examples. To improve the coding reliability a coding book was then build, based on the enhancements made to the initial coding scheme following the review and discussion. It includes definitions and coding rules that help coders to converge to a consistent coding. These rules aimed at facilitating the coding, while being in line with the main analysis objectives (co-creativity assessment). Here are some examples of such rules:

- Prioritize clients interactions when designer and client talk at the same time,

- Even short interventions, just made of interjection such as 'OK' or 'Yeah', have to be coded,

- Prioritize ephemeral artefact comparatively to others types of artefacts, meaning that we code Ephemeral when an actor gestures around a Tangible, Digital or Mixed artefact.

At the end of the process, the Cohen's Kappa associated to the coding of a new video period of 5 minutes by the same two coders were:

Table 1. Cohen's Kappa for each session analysis

\begin{tabular}{|c|c|c|c|c|c|c|}
\hline & \multicolumn{2}{|c|}{ AR session } & \multicolumn{2}{|c|}{ SAR session } & \multicolumn{2}{|c|}{ Standard session } \\
\hline On The Fly & Cohen's Kappa & $\%$ agreement & Cohen's Kappa & $\%$ agreement & Cohen's Kappa & $\%$ agreement \\
\hline Actor & 0,58 & 76 & 0,71 & 84 & 0,55 & 73 \\
\hline Artefact & 0,30 & 49 & 0,59 & 71 & 0,62 & 74 \\
\hline Post session & 0,59 & 68 & 0,45 & 59 & 0,50 & 64 \\
\hline
\end{tabular}


If we refer to the Cohen's Kappa scale (Landis and Koch, 1977), the results show substantial agreement (green), moderate agreement (yellow) and fair (red). We can see a reduced agreement level on the first excerpt of the AR session with the Artefact coding $(0,30)$. Artefact real time coding is the most difficult to grasp and requires substantial training. However, we considered the level of convergence sufficient as the two other samples show moderate to substantial agreement (SAR and Standard sessions) level. To further improve inter-coder agreement, some rules have been defined to prevent the overload of the coder with repetitive events. For example, we decided not to code the reiteration of the same kind of interaction made after each other by the same actor. This might apply when a designer is interacting, stops speaking, and then re-initiates an interaction - the actors' coder will not code twice the designer. However, if two different designers carry on over each other, this will lead to several occurrences of the same actor type.

Once we have verified that the coding on the fly is doable and reliable, we end up with two separated coding. Actually, two files in csv format include two columns:

- The timestamp of the recorded events

- The nature of the events (type of actor in one file, type of artefact in the other).

With these two files prepared, we can proceed with the data processing.

\subsubsection{Data processing procedure}

Our objective at this point in the process is to merge the results of actors and artefacts coding into a single file that faithfully represents the interactions happening during the session, at least from a quantitative point of view.

One issue here was how to deal with the inconsistency of the timing between the two files. Even though the files are synchronized before merging the data, the researchers when coding might react within a lapse of time that could generate events delayed of 1 to 2 seconds. For example, in Figure 5 at the timestamp 05:44 Client is quoted and Digital is quoted on minute 05:43. When the event occurs at a 'quiet' moment, isolated from others interactions, there's no doubt about the fact that coders coded the same interaction, despite the fact that they code the events with slightly different time stamps. In this case, it was logical to define a rule stating that we accept a positive or negative offset of one second in the coding of the same interaction. Hence, in the previous example, we quoted Client digital in the fourth column that represents the coding resulting from our method. The result is more uncertain when several events are coded in a short interval (sometimes in the same second). Then, the chronology of the recorded events is essential to associate both part of the code.

Another issue is due to the fact that we were only coding occurrences (actually the beginning of interactions). Nevertheless, interactions are continuous. We consider that until another actor intervenes, the current actor (the last identified) still interacts. As an example (Figure 5), at 05:49, Designer interaction was tracked, and made use of an Ephemeral artefact. At 05:51 a new artefact (a Mixed artefact) has been tracked. In application of the coding rule stating that we do not code again if the actor is still the same, we jump back to the previous actor's event (05:49) to quote on line 05:51 as 'D Mixed' in the fourth column. The same rule applies to the event coded at 05:56 and 05:58. A Mixed artefact has been spotted at 05:51 and no other different artefact has been tracked until 05:58. Hence, we consider that Client interacted at 05:56 on the mixed prototype, and same goes for the Designer at 05:58, ending up with a Designer Mixed ('D Mixed') interaction.

An exception is made to the rule described above. It concerns a certain category of artefact. We consider that an Ephemeral artefact is attached to the actor that is staging it. Hence, the event of Ephemeral artefact is not replicated when occurs a change of actors without any artefact coded. Consequently, if there is no other artefact coded it should be coded against the 'None' category. (meaning that the actors had a spoken interaction without any identified artefact). Figure 6 illustrates this rule. At 07:36, a Designer is quoted with Ephemeral artefact (fourth column). At 07:38, a Designer intervenes. No new artefact is coded. According to the previous rule we do not quote Designer Ephemeral but Designer None. The same logic applies to the Client interaction quoted 'None' at 07:38. These different rules we have developed are included in a set of 'merging rules' that make explicit a common set of heuristics. These rules help to balance the effect of splitting the coding. 


\begin{tabular}{|c|c|c|c|}
\hline 00:05:41 & Designer & Ephemeral & D Ephemeral \\
\hline 00:05:42 & Designer & Ephemeral & D Ephemeral \\
\hline 00:05:43 & & Digital & \\
\hline 00:05:44 & Client & & C Digital \\
\hline 00:05:45 & & & \\
\hline 00:05:46 & & Ephemeral & C Ephemeral \\
\hline 00:05:47 & & Mixed & C Mixed \\
\hline 00:05:48 & & & \\
\hline 00:05:49 & Designer & Ephemeral & D Ephemeral \\
\hline $00: 05: 50$ & & & \\
\hline 00:05:51 & & Mixed & D Mixed \\
\hline 00:05:52 & & & \\
\hline 00:05:53 & & & \\
\hline 00:05:54 & & & \\
\hline 00:05:55 & & & \\
\hline 00:05:56 & Client & & C Mixed \\
\hline 00:05:57 & & & \\
\hline 00:05:58 & Designer & & D Mixed \\
\hline
\end{tabular}

Figure 5. Merged table sample 1

\begin{tabular}{|c|c|c|c|}
\hline 00:07:28 & & Ephemeral & \\
\hline 00:07:29 & Designer & & D Ephemeral \\
\hline \multicolumn{4}{|l|}{ 00:07:30 } \\
\hline \multicolumn{4}{|l|}{ 00:07:31 } \\
\hline 00:07:32 & & None & D None \\
\hline \multicolumn{4}{|l|}{ 00:07:33 } \\
\hline 00:07:34 & & Mixed & D Mixed \\
\hline \multicolumn{4}{|l|}{ 00:07:35 } \\
\hline 00:07:36 & & Ephemeral & D Ephemeral \\
\hline \multicolumn{4}{|l|}{ 00:07:37 } \\
\hline 00:07:38 & Designer & & D None \\
\hline 00:07:38 & Client & & C None \\
\hline
\end{tabular}

Figure 6. Merged table sample 2

Our testing of the method on multiple samples of video lead us to the conclusion that with our coding book, complemented with the 'set of rules' we generated, it is possible to code on the fly in a reliable manner using the method we proposed. In the following section, we examine the validity of the coding obtained by this method in more detail.

\section{Validation of the method}

To define the validity of the proposed method, we applied it to the coding of three sessions carried out under experimental conditions in our laboratory in collaboration with our industrial partner, which we will refer to as 'Company S'. The results we obtained were then compared to those obtained by applying the traditional post session method that we will present hereafter (section 4.2).

\subsection{Design session}

Our analysis was based on sessions that lasted around 35 minutes and involved two Designers from Company $\mathrm{S}$ and three potential end users. The designers were the same for the three sessions however, the end users were different for each session. The designed product was different for each session. Each of them was proposed by the designers of Company $\mathrm{S}$ who freely managed the session as they planned. Design representation tools available were specific for each session: an Augmented Reality (AR) tool for the session 1 (see Figure 7), a Spatial Augmented Reality (SAR) tool (SPARK) for the session 2 (see Figure 8), and standard session without specific tool for the last session.

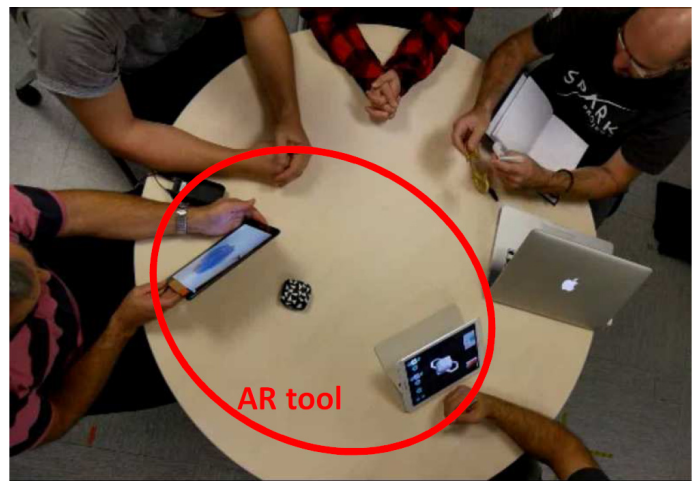

Figure 7. AR tool in use

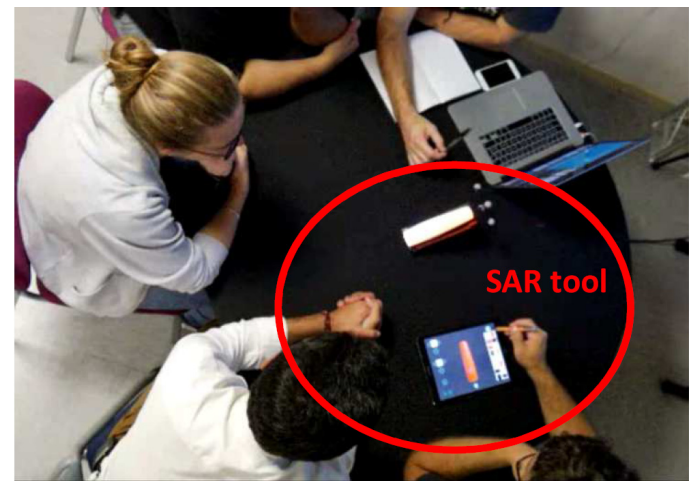

Figure 8. SAR tool in use

Each session began by a short presentation, made by the designers using PowerPoint slides, about the product being designed. Then a co-creative session was initiated to make the proposals evolve in light of suggested ideas while using means available for the session (AR tool, SAR tool or without specific tool). 
For this paper, as cited in the introduction, our research question is a methodological one: does a coding on the fly give us comparable quantitative results to a post session coding?

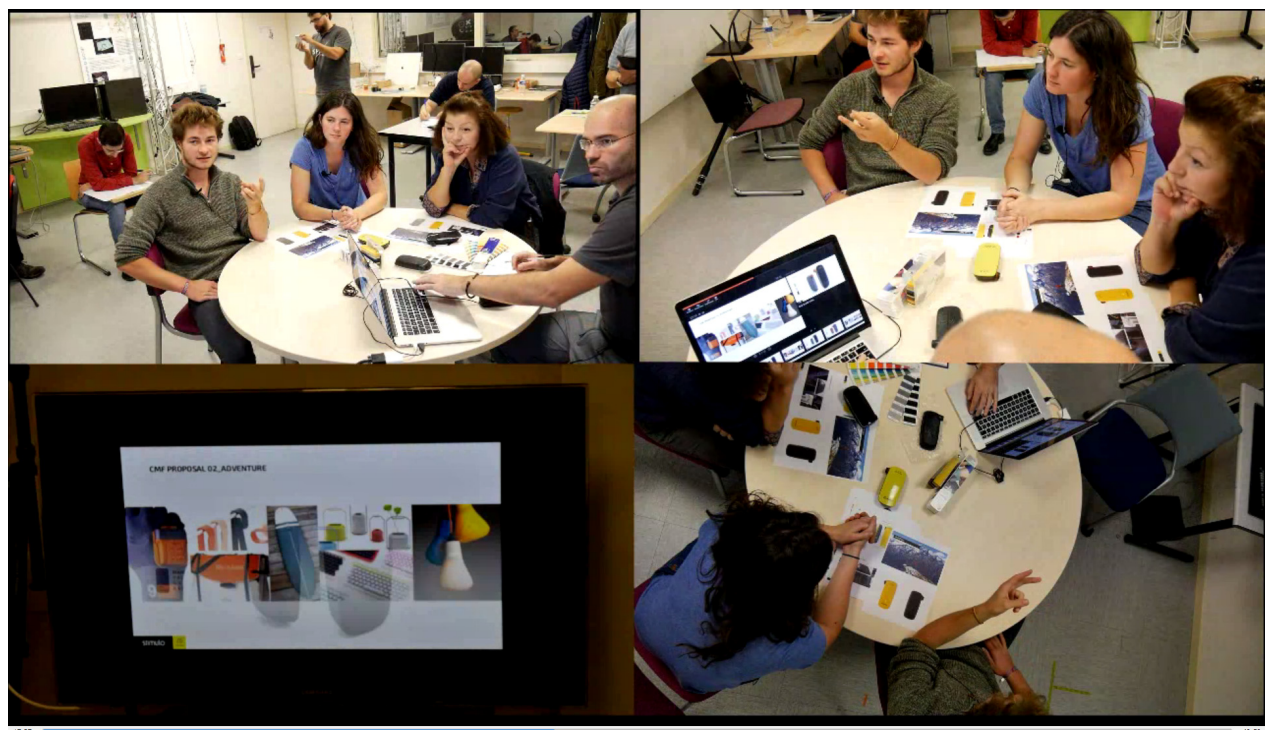

Figure 9. A screen shot of the processed video from standard session

\subsection{Post session coding}

To answer our research question, the method traditionally used is based on post session coding. A video and audio capture of the design scene has to be made as basic material for this coding. Regarding the visual aspect, we used four cameras that recorded four points of view to catch as much information as possible. Regarding the audio aspect, each actor was equipped with a lapel microphone. Audio and video information were then processed and integrated into a file that could be watched using a multimedia player. A screen shot of this integration is showed below (Figure 9). A coding was then made by researchers using the video file and supported by the coding scheme we describe in section 2 plus an Excel file. The latter includes in the first column time incremented every second and in the second and third columns a description of the event that occurs at this time by qualifying actor that intervene and artefact used to support his interaction (see Figure 10).

\begin{tabular}{|r|l|l|}
\hline Time code & \multicolumn{1}{|c|}{ Actor } & \multicolumn{1}{|c|}{ Artefact } \\
\hline 00:06:03 & & \\
\hline $00: 06: 04$ & Designer & Ephemeral \\
\hline $00: 06: 05$ & & Mixed \\
\hline $00: 06: 06$ & & $\begin{array}{l}\text { Digital } \\
\text { Tangible } \\
\text { Ephemeral } \\
\text { None }\end{array}$ \\
\hline 00:06:07 & & Tangible \\
\hline $00: 06: 08$ & Client & Tangible \\
\hline $00: 06: 09$ & Designer & \\
\hline $00: 06: 10$ & & Digital \\
\hline $00: 06: 11$ & Designer & \\
\hline $00: 06: 12$ & & \\
\hline $00: 06: 13$ & Client & Digital \\
\hline $00: 06: 14$ & & \\
\hline
\end{tabular}

Figure 10. Post session coding result

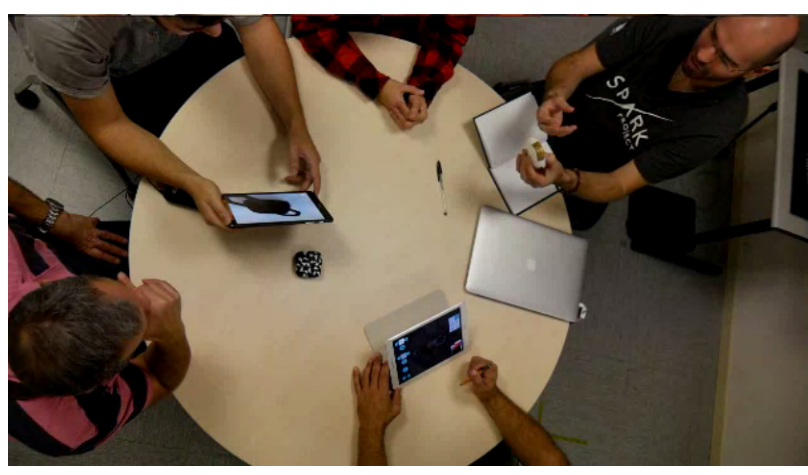

Figure 11. Designer gesturing using an ephemeral artefact

To ensure the reliability of this coding, a double coding was carried out by two researchers. This led to a post session coding book that allows the Cohen Kappa to go up to 0,8 . 


\subsection{Comparison of results obtained between post session coding and coding on the fly}

From coding made using both methods (on the fly and post session), we processed data in order to have deeper information about who initiate an intervention, and what kind of artefact is used. The objective here is not to discuss the nature of the interactions, but to compare results obtained using the two alternative methods. For this comparison, we focused on two kinds of information:

- percentage of actors' interventions obtained by each method,

- percentage of interactions quoted for each category of the scheme.

Table 2 shows that the results concerning the type of actor initiating the intervention (Client vs Designer) are very close for the AR and SAR conditions, but show a significant difference for the standard condition.

Table 2. The percentage of designers and clients interactions

\begin{tabular}{|l|c|c|c|c|}
\hline & \multicolumn{2}{|l|}{ \% of Designers interaction } & \multicolumn{2}{l|}{ \% of Clients interaction } \\
\hline & On the fly & Post Processing & On the fly & Post Processing \\
\hline Standard conditions & 57,2 & 49,3 & 42,8 & 50,7 \\
\hline SAR conditions & 66,9 & 67,2 & 33,1 & 32,8 \\
\hline AR Conditions & 63,9 & 63,6 & 36,1 & 36,4 \\
\hline
\end{tabular}

Tables hereafter shows for each session a visual representation of percentage of interactions quoted for each category of the scheme on the left from the 'on the fly' coding method and on the right results from post session coding method.

Table 3. Interactions during Augmented Reality (AR) session

\begin{tabular}{|c|c|c|c|c|c|}
\hline & & \multicolumn{4}{|c|}{ AR Session } \\
\hline & & \multicolumn{2}{|c|}{ ON THE FLY } & \multicolumn{2}{|c|}{ POST-SESSION } \\
\hline & & Designer & Client & Designer & Client \\
\hline \multirow[t]{4}{*}{ Artefacts } & Digital & $42 \%$ & $27 \%$ & $42 \%$ & $26 \%$ \\
\hline & Tangible & $4 \%$ & $2 \%$ & $2 \%$ & $2 \%$ \\
\hline & Ephemeral & $12 \%$ & $4 \%$ & $14 \%$ & $5 \%$ \\
\hline & None & $6 \%$ & $3 \%$ & $6 \%$ & $3 \%$ \\
\hline
\end{tabular}

Table 4. Interactions during Spatial Augmented Reality (SAR) session

\begin{tabular}{|c|c|c|c|c|c|}
\hline & \multicolumn{4}{|c|}{ SAR Session } \\
\hline & & \multicolumn{2}{|c|}{ ON THE FLY } & \multicolumn{2}{|c|}{ POST-SESSION } \\
\hline & & Designer & Client & Designer & Client \\
\hline \multirow[t]{4}{*}{ Artefacts } & Mixed & $38 \%$ & $20 \%$ & $37 \%$ & $19 \%$ \\
\hline & Digital & $2 \%$ & $0 \%$ & $3 \%$ & $0 \%$ \\
\hline & Ephemeral & $16 \%$ & $8 \%$ & $15 \%$ & $5 \%$ \\
\hline & None & $11 \%$ & $5 \%$ & $13 \%$ & $8 \%$ \\
\hline
\end{tabular}

Table 5. Interactions during Standard session

\begin{tabular}{|c|c|c|c|c|c|}
\hline & \multicolumn{4}{|c|}{ STANDARD Session } \\
\hline & & \multicolumn{2}{|c|}{ ON THE FLY } & \multicolumn{2}{|c|}{ POST-SESSION } \\
\hline & & Designer & Client & Designer & Client \\
\hline \multirow[t]{4}{*}{ Artefacts } & Digital & $17 \%$ & $15 \%$ & $14 \%$ & $18 \%$ \\
\hline & Tangible & $15 \%$ & $12 \%$ & $13 \%$ & $16 \%$ \\
\hline & Ephemeral & $18 \%$ & $10 \%$ & $15 \%$ & $7 \%$ \\
\hline & None & $7 \%$ & $6 \%$ & $8 \%$ & $9 \%$ \\
\hline
\end{tabular}


Results concerning the percentage of each category of interaction seem also to be very close and give us positive argument to consider that an 'on the fly' coding approach is accurate. From these data, we can conclude that the method we proposed provided results that can be considered as quite relevant. Moreover, these results are made available after the end of the session in a much shorter time. If we do not take into account, on one hand the duration of the session itself, and on the other hand the necessary time for quantitative analyses made from the encodings in Excel files that are common to both methods, the necessary time to do the coding of a 30 minutes section of a session is three time shorter for the on the fly approach compared to the traditional, post-session coding approach.

Table 6. Time taken to complete the coding of a 30 minutes section of a session using the on the fly vs traditional coding approach

\begin{tabular}{|l|l|l|}
\hline & Traditional post session coding & On the fly coding \\
\hline Time to process the video file & $2 \mathrm{~h}$ & -- \\
\hline Time to process & $4 \mathrm{~h}$ & $2 \mathrm{~h}$ (merging) \\
\hline Total & $6 \mathrm{~h}$ & $2 \mathrm{~h}$ \\
\hline
\end{tabular}

\section{Conclusion}

The purpose of the paper is to present a method which provide a quick quantitative evaluation of gestural artefact-centric interactions made by designers and clients during a co-creative design session. To reach this aim, we created an on the fly method, that allows real time capture and fast processing of a data set. In order to check the reliability of this method, we have first validated the on the fly procedure with two coders and created a coding book. We have reached a significant agreement measured with a Cohen's Kappa index. Second we compared results obtained by applying the 'on the fly' method and the traditional post-session coding method to a set of three experimental sessions. The elements of comparison were percentage of actors' interventions obtained by each method and percentage of interactions quoted for each category of our predefined coding scheme. The preliminary results show that this approach is accurate and offers a considerable time savings with respects to the traditional coding approach. This method also allows identifying key moments that will further be encoded in order to analyse micro-activities with conventional approaches.

\section{Acknowledgment}

The work reported in this paper is part of the SPARK project, funded by the European Union's Horizon 2020 research and innovation programme under grant agreement No.688417. This paper reflects only the authors' views and the European Commission is not responsible for any use that may be made of the information it contains. The authors would like to thank all the partners of the SPARK Consortium and especially Maud Poulin and Céline Gros for their extensive support and valuable contribution to the development of this research.

\section{References}

Ardito, C., Buono, P., Costabile, F., Lanzilotti, R. and Piccinno, A. (2012), "End users as co-designers of their own tools and products", Journal of Visual Languages and Computing, Vol. 23 No. 2, pp. 78-90.

Austin, J.L. (1962), How to do things with words, Harvard University Press, Boston. https://doi.org/10.1016/j.jvlc.2011.11.005

Becattini, N., Masclet, C., Ben Guefrache, F., Prudhomme, G., Cascini, G. and Dekoninck, E. (2017), "Characterisation of a co-creative design session through the analysis of multi-modal interactions", Proceedings of the International Conference on Engineering Design (ICED 2017), Vancouver, Canada, August, 21-25, 2018, The Design Society, pp. 479-488.

Cash, P. and Maier, A. (2016), "Prototyping with your hands: the many roles of gesture in the communication of design concepts", Journal of Engineering Design, Vol. 27 No. 1-3, pp. 118-145. https://doi.org/10.1080/09544828.2015.1126702

Cash, P. and Snider, C. (2014), "Investigating design: A comparison of manifest and latent approaches", Design Studies, Vol. 35 No. 5, pp. 441-472.

Cross, N., Christiaans, H. and Dorst, K. (1996), Analysing design activity, Wiley, Chichester.

Davis, B. (2016), "Gesture, creativity and design", Proceedings of the fourth International Conference on Design Creativity, Georgia Institute of Technology, Atlanta, November 2-4, 1996. 
Ericsson, K.A. and Simon, H.A. (1993), Protocol Analysis: Verbal Reports as Data, Bradford Books, MIT Press, London.

Eris, O., Martelaro, N. and Badke-Schaube, P. (2014), “A comparative analysis of multimodal communication during design sketching in co-located and distributed environments", Design Studies, Vol. 35 No. 6, pp. 559592. https://doi.org/10.1016/j.destud.2014.04.002

Gero, J.S. and Mc Neill, T. (1998), “An approach to the analysis of design protocols”, Design Studies, Vol. 19 No. 1, pp. 21-61. https://doi.org/10.1016/S0142-694X(97)00015-X

Kendira, A., Gidel, T., Jones, A., Lenne, D., Barthes, J.-P. and Moulin, C. (2011), "Conducting Preliminary Design around an Interactive Tabletop", Proceedings of the 18th International Conference on Engineering Design (ICED 2011), pp. 366-376.

Landis, J. and Koch, G. (1977), “The measurement of observer agreement for categorical data”, Biometrics, Vol. 33 No. 1, pp. 159-174. https://doi.org/10.2307/2529310

McNeill, D. (1992), Hand and Mind: What Gestures Reveal about Thought, University of Chicago Press, Chicago.

Thomas, K. (2000), “Collaborative design: what is it?”, Automation in Construction, Vol. 9 No. 4, pp. 409-415. https://doi.org/10.1016/S0926-5805(99)00025-4

Visser, W. (2010), "Function and form of gestures in a collaborative design meeting", In: Kopp, S. and Wachsmuth, I. (Eds.), Gesture in embodied communication and human-computer interaction, 8th international gesture workshop, Bielefeld, Germany: Springer Berlin Heidelberg, pp. 61-72. https://doi.org/10.1007/978-3642-12553-9 6

Visser, W. and Maher, M.L. (2011), "The role of gesture in designing”, Artificial Intelligence for Engineering Design, Analysis and Manufacturing (AIEDAM), Vol. 25 No. 3, pp. 213-220. https://doi.org/10.1017/S0890060411000047

Fatma Ben Guefrache, PhD Student

Université Grenoble Alpes, Institut polytechnique de Grenoble, Industrial Engineering

46 avenue de Felix viallet, 38031 Grenoble, France

Email: fatma.ben-guefrache@g-scop.grenoble-inp.fr 